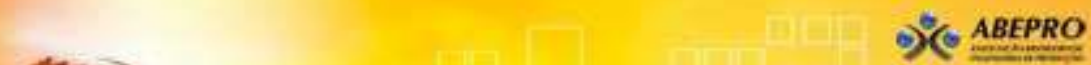 PRODUCIF̄O ONLINE REVISTA CIENTIFICA ELETRONICA DE ENGENHARIA DE PRODUCAO ISSN 1676-1901
}

\section{GRÁFICOS DE CONTROLE: ASPECTOS TEÓRICOS E PRÁTICOS A PARTIR DA LÓGICA FORMAL E DA LÓGICA FUZZY}

\section{CONTROL CHARTS: THEORETICAL AND PRACTICAL ASPECTS FROM THE FORMAL LOGIC AND FUZZY LOGIC}

\author{
Ismael Batista Maidana Silvestre* E-mail: silvestreismaelil@yahoo.com.br \\ Viviane Leite Dias de Mattos** E-mail: viviane.leite.mattos@gmail.com \\ Catia Maria dos Santos Machado** E-mail: catiamachado.furg@gmail.com \\ *Instituto Federal de Educação, Ciência e Tecnologia Farroupilha (IFFarroupilha), Alegrete, RS \\ **Universidade Federal do Rio Grande (FURG), Rio Grande, RS
}

\begin{abstract}
Resumo: No monitoramento de variáveis complexas, subjetivas ou mal definidas, alguns processos produtivos podem ser avaliados a partir de informações imprecisas, razão pela qual é importante buscar estratégias que viabilizem a análise e interpretação desses dados. $O$ presente artigo apresenta uma estratégia para construção de gráficos de controle fuzzy, envolvendo também um método para transformar valores crisp em valores fuzzy e um método para ordenar números fuzzy. Os gráficos obtidos são comparados com os tradicionais gráficos de controle de Shewhart em um contexto de informações imprecisas: o estudo de algumas características sensoriais definidoras da qualidade de um produto em uma indústria de alimentos (cor, aroma, consistência, sabor e acidez). Foi constatado que os gráficos de controle fuzzy apresentaram maior flexibilidade, com a criação de zonas de alerta internas e externas, sendo menos rigorosos na avaliação da tendência central e um pouco mais rigorosos na avaliação da variabilidade, quando usado um grau de dominância maior.
\end{abstract}

Palavras-chave: Qualidade. Gráficos de Controle. Lógica Fuzzy. Processo de Produção.

Abstract: Many processes are analyzed from vague or imprecise information. It is important to find strategies that enable the analysis and interpretation of such data. This article presents a strategy for building fuzzy control charts, involving a method for transforming crisp values into fuzzy values and a method for ordering fuzzy numbers. The charts obtained are compared with the traditional Shewhart control charts in a context of inaccurate: the study of some sensory characteristics of a product in a food industry (color, aroma, texture, taste and acidity). It was found that the fuzzy control charts showed more flexibility, with the creation of internal and external warning areas. They are less rigorous in evaluating the central tendency and a little more rigorous in assessing the variability.

Keywords: Quality. Control Charts. Fuzzy Logic. Production Process.

\section{INTRODUÇÃO}

Segundo Besterfield (1995), a norma A3-1987 ANSI/ASQC define qualidade como a totalidade de requisitos e características de um produto ou serviço que estabelecem a sua capacidade de satisfazer determinadas necessidades do consumidor. De acordo com Mello (2011), Deming complementa esse conceito ressaltando que a qualidade pode ser medida por meio da interação entre o produto, Revista Produção Online, Florianópolis, SC, v. 16, n. 2, p. 754-778, abr.jun. 2016. 
o cliente e o atendimento a esse cliente. Qualidade significa, então, atender continuamente as necessidades dos clientes, a um preço que eles estejam dispostos a pagar. De acordo com Moller (1999), apud Silva, Medeiros e Costa (2009), investimentos na área da qualidade permitem não apenas a melhoria do produto ou serviço, mas também da imagem da empresa, de sua posição financeira, do ambiente de trabalho e da satisfação dos clientes, entre outros.

Portanto, para se manter em um mercado competitivo e em constante mudança, as empresas buscam cada vez mais alternativas para melhorar a qualidade dos produtos ofertados, visando atender as exigências dos consumidores, garantindo, dessa forma, a sua fidelização. Parte-se do pressuposto de que o que garante a sobrevivência de uma empresa nesse mercado exigente é ter produtos com boa qualidade e custo competitivo. Nesse contexto a estatística, ou ainda, as técnicas de controle estatístico de processos (CEP), como os gráficos de controle, vêm sendo muito usadas com o objetivo de monitorar e caracterizar processos, buscando melhorar a qualidade e diminuir custos associados às falhas de processo. Nesse caso, ao final do processo, é possível obter produtos com maior qualidade, o que vai ao encontro do esperado pelos consumidores.

As normas da ISO 9000 (International Organization for Standardization) indicam a utilização do CEP em seu controle de qualidade. As empresas certificadas ou em processo de certificação precisam implantar o controle estatístico em seu processo produtivo. Trata-se de uma estratégia preventiva de controle de qualidade que possibilita uma avaliação do processo em tempo real. Dessa forma, diminui a quantidade de produtos defeituosos no final do processo.

Segundo Montgomery (2012), os gráficos de controle, muito usados em CEP, são uma das ferramentas mais importantes para estabilização de processos industriais. Além de serem de fácil entendimento, possíveis problemas no processo podem ser facilmente visualizados de forma que, muitas vezes, o próprio operador pode identificar um comportamento fora dos padrões pré-estabelecidos.

Muitos gráficos de controle já foram desenvolvidos e tiveram sua eficiência comprovada. Entretanto, eles têm sido construídos partindo do pressuposto de que as informações são precisas, o que muitas vezes pode não acontecer. Esse é o caso das características sensoriais, muito utilizadas na indústria de alimentos e que dependem da percepção dos indivíduos. A teoria da lógica fuzzy parece ser bastante útil nesse contexto, pois de acordo com Zadeh (1975), ela é especialmente indicada Revista Produção Online, Florianópolis, SC, v. 16, n. 2, p. 754-778, abr./jun. 2016. 
na mensuração de variáveis complexas ou mal definidas, por possibilitar a diminuição da ocorrência de resultados incorretos. Ela se fundamenta no princípio da incompatibilidade: alta precisão é incompatível com alta complexidade. A aceitação dessa premissa sugere que para poder fazer avaliações sensoriais, por exemplo, pode ser necessário o abandono de elevados padrões de rigor e precisão. Essa estruturação é mais semelhante à maneira de pensar e tirar conclusões dos humanos.

A avaliação sensorial está ligada com a resposta humana a estímulos físicos e pretende quantificar a resposta de indivíduos frente a um estímulo. Civille e Oftedal (2012) fazem uma síntese da evolução das técnicas de análise sensorial, considerando que elas se concentram tanto na medida objetiva de propriedades sensoriais dos produtos (compreender o produto) e nas respostas subjetivas dos indivíduos (compreensão dos consumidores), bem como na interpretação da resposta do consumidor através da compreensão da resposta ao produto (ligando produto e atendimento ao consumidor).

O objetivo deste estudo é apresentar uma estratégia para construção de gráficos de controle fuzzy. Para tanto, é necessário definir um método para gerar e outro para ordenar números fuzzy. Seu desempenho é analisado por meio da comparação com os tradicionais gráficos de controle de Shewhart em um contexto de informações imprecisas: o estudo de algumas características sensoriais definidoras da qualidade de um produto em uma indústria de alimentos.

\section{REVISÃO DE LITERATURA}

Em um processo produtivo, as diversas características definidoras da qualidade dos produtos sempre apresentam variabilidade, pois nunca dois itens são exatamente iguais. De acordo com Samohyl (2009), melhorando a qualidade desse processo, essa variabilidade pode ser reduzida tornando-se infinitesimal, mas jamais será eliminada. Segundo Besterfield (1995), ela pode ter sua origem associada a dois tipos de causas: as comuns, intrínsecas ao processo e que não podem ser eliminadas, e as especiais, que podem ser identificadas e eliminadas. Tratar uma causa comum como sendo especial gera custos desnecessários e maior variabilidade na produção. Por outro lado, se causas especiais passarem 
despercebidas, podem ser incorporadas ao processo, perdendo-se oportunidade de melhoria do produto.

Para identificar e diferenciar essas causas, o Dr. Walter Shewhart desenvolveu uma técnica simples, porém muito eficaz, chamada gráfico de controle de processo. A partir de então, surgiram vários tipos de gráficos de controle, sendo seu emprego associado ao tipo de variável a ser analisada.

Para Franco et al. (2014), os gráficos de controle de Shewhart são procedimentos simples utilizados para monitoramento de variabilidade em processos que pretendem atingir elevados padrões de qualidade e manter em níveis aceitáveis o número de itens não conformes. Mediante a utilização correta de gráficos de controle é possível identificar rapidamente alterações em pontos estratégicos de linhas de produção.

Já Samohyl (2009), considera os gráficos de controle como elementos visuais extremamente úteis para o monitoramento da conformidade de características dos produtos e processos. Atualmente, empresas de vários setores da economia percebem as vantagens da utilização dos gráficos de controle e, um número considerável dessas, os utiliza com o objetivo de diminuir perdas e melhorar a qualidade do produto final.

O gráfico de controle descreve o processo considerando duas características fundamentais: centralização, normalmente determinada pela média, e dispersão, geralmente verificada pelo desvio-padrão ou amplitude.

Em linhas gerais, os tradicionais gráficos de controle de Shewhart são compostos por três linhas: uma linha central, que representa a centralização do processo, e duas outras linhas associadas aos seus limites: um inferior e outro superior. De acordo com Montgomery (2012), o uso dos três desvios-padrão tem funcionado bem, mas por vezes alguns analistas sugerem que o uso de outros critérios para os limites de controle pode ser conveniente.

$\mathrm{Na}$ interpretação desses gráficos deve-se priorizar a busca por padrões, por evidências de que o processo esteja sob controle estatístico. Assim, se todos os pontos plotados no gráfico estiverem entre os dois limites de controle e sem qualquer tendência particular, ou seja, apresentando uma disposição aleatória, encontram-se evidências de que o processo está sob controle estatístico. Quando a distribuição dos dados apresentar graficamente algum comportamento atípico ou não aleatório, isso pode ser considerado como sintoma de causa especial que esteja Revista Produção Online, Florianópolis, SC, v. 16, n. 2, p. 754-778, abr./jun. 2016. 
ocorrendo no processo de produção. Fazem parte desses padrões: periodicidade ou ciclos, tendência, deslocamento da tendência central, falta ou excesso de variabilidade e sequências, entre outros. Cálculos probabilísticos podem ser muito úteis no reconhecimento desses padrões. Mais detalhes podem ser encontrados em Trip e Does (2010).

Alguns autores, entretanto, têm evidenciado 0 fato desses gráficos convencionais (com base nos limites de três sigmas) apresentarem excesso de falsos alarmes, como Joekes, Smrekar e Barbosa (2015), que avaliaram o uso de gráficos para proporção de não-conformes em pequenas amostras no monitoramento de processos de alta qualidade. Além disso, sob o enfoque da lógica formal, os diversos tipos de gráficos de controle consideram que todas as mensurações não apresentam erro. Sentürk et al. (2014), entretanto, chamam atenção para o fato dos dados das amostras utilizadas em gráficos de controle poderem incluir incertezas provenientes de sistemas de medição ou de variações ambientais e que, números fuzzy ou variáveis linguísticas podem ser usados para capturar essas incertezas. Por outro lado, na indústria de alimentos, a atribuição de notas às características sensoriais por um grupo de juízes é uma prática bastante frequente na avaliação de características definidoras da qualidade. Diante do que foi exposto, parece ser muito pertinente utilizar essas notas para gerar um número fuzzy que represente o resultado final da característica avaliada.

loannou et al. (2002), por exemplo, propõem uma abordagem baseada na teoria dos conjuntos fuzzy para avaliar a ocorrência de um defeito em salsichas (formação de crostas), cuja avaliação depende da percepção do avaliador. O modelo proposto levou em consideração a percepção de dois avaliadores e um banco de dados com 76 imagens. Os autores consideram que os conceitos matemáticos utilizados no trabalho parecem promissores, mas aconselham o desenvolvimento de outros trabalhos comparando mensurações que dependam da percepção com mensurações que dependam de instrumentos.

Kupongsak e Tan (2006) também usaram números fuzzy, além de redes neurais, para alcançar a qualidade desejada para atributos sensoriais de alimento oriundo de um processo industrial (bolo de arroz). Os números fuzzy foram usados para interpretar respostas sensoriais e, segundo os autores, o estudo desenvolvido demonstrou o grande potencial dessa teoria para quantificar as avaliações 
sensoriais, salientando, entretanto, que mais pesquisas são necessárias para determinar a eficácia geral da abordagem para outros produtos e processos.

\subsection{Gráficos de controle fuzzy}

$\mathrm{Na}$ construção de gráficos de controle fuzzy três aspectos devem ser considerados: a geração de números fuzzy utilizados na mensuração das características de qualidade, a construção do gráfico de controle propriamente dito e sua interpretação, que requer a utilização de um método de ordenação, tarefa não muito simples quando se trata de números fuzzy.

\subsubsection{Geração de números fuzzy}

Cheng (2005) apresenta um método de geração de números fuzzy a partir de um conjunto de valores discretos, descrito resumidamente a seguir:

Sejam as observações, $g_{i}$, onde $i=1,2,3, \ldots, n$, sendo $n$ a quantidade de observações considerada. Inicialmente são calculadas as distâncias entre as observações de acordo com a expressão (1), organizadas em uma matriz com $i$ linhas e $j$ colunas $(j=1,2,3, \ldots, n)$, e suas respectivas proximidades médias ao centro das obervações, conforme expressão (2). O grau de importância de cada observação é fornecido pela expressão (3) e o centro do número fuzzy é determinado pela expressão (4) (CHENG, 2005).

$$
\begin{gathered}
d_{i j}=\left|g_{i}-g_{j}\right| \\
\bar{d}_{i}=\sum_{j=1}^{n} \frac{d_{i j}}{(n-1)} \\
w_{j}=\frac{1}{\sum_{i=1}^{n} p_{i j}}, j=1, \ldots, n, \text { onde } p_{i j}=\frac{\bar{d}_{j}}{\bar{d}_{i}} \\
m=\sum_{i=1}^{n} w_{i} g_{i}
\end{gathered}
$$

Para estimar a extensão do número fuzzy, isto é, encontrar seus limites, inferior e superior, inicialmente determina-se a variabilidade das possibilidades, 0 que é feito de acordo com a expressão (5) (CHENG, 2005). 


$$
\hat{S}^{2}=\frac{1}{2}\left\{\frac{\sum_{i \in \arg \left\{g_{i}<m\right\}} w_{i}\left(m-g_{i}\right)^{2}}{\sum_{i \in \arg \left\{g_{i}<m\right\}} w_{i}}+\frac{\sum_{i \in \arg \left\{g_{i}>m\right\}} w_{i}\left(m-g_{i}\right)^{2}}{\sum_{i \in \arg \left\{g_{i}>m\right\}} w_{i}}\right\}
$$

Após, calculam-se $g^{l}$ e $g^{r}$ como a média ponderada das observações menores que $m$ e a média ponderada das observações maiores que $m$, o que é feito, respectivamente, pelas expressões (6) e (7). Esses resultados são utilizados para encontrar a razão de distribuição dos dados da esquerda para a direita, o que é feito pela expressão (8). Finalmente, os limites $a$ e $b$ são encontrados pelas expressões (9) e (10) (CHENG, 2005).

$$
\begin{array}{r}
g^{l}=\frac{\sum_{i \in \arg \left\{g_{i}<m\right\}} w_{i} g_{i}}{\sum_{i \in \arg \left\{g_{i}<m\right\}} w_{i}} \\
g^{r}=\frac{\sum_{i \in \arg \left\{g_{i}>m\right\}} w_{i} g_{i}}{\sum_{i \in \arg \left\{g_{i}>m\right\}} w_{i}} \\
\rho=\frac{m-g^{l}}{g^{r}-m} \\
a=m-\sqrt{\frac{12 \rho^{2} s^{2}}{1+\rho^{2}}} \\
b=m+\sqrt{\frac{12 s^{2}}{1+\rho^{2}}}
\end{array}
$$

Mais detalhes sobre este método podem ser encontrados em Cheng (2005).

Com os parâmetros $m, a$ e $b$, o número fuzzy triangular, denotado como $(a, m, b)$, é definido conforme expressão (11), onde $0 \leq g \leq G$, sendo $G$ o maior valor possível atribuído a uma observação.

$$
\mu(g ; a, m, b)=\left\{\begin{array}{cl}
1-\frac{m-g}{m-a}, & a \leq g \leq m \\
1-\frac{g-m}{b-m}, & m<g \leq b \\
0, & \text { outros valores }
\end{array}\right.
$$

\subsubsection{Construção de gráficos de controle fuzzy}

Os gráficos de controle propostos em Shu e Wu (2011) são, de certa forma, similares aos tradicionais gráficos de controle Shewhart no sentido de considerarem Revista Produção Online, Florianópolis, SC, v. 16, n. 2, p. 754-778, abr.jjun. 2016. 
seus limites de controle a três desvios padrão da linha central. Similarmente, também são construídos dois gráficos: um para monitorar a média $(\mu)$ e outro para monitorar a variabilidade, este último utilizando a amplitude $(R)$. Na construção de ambos, descritas a seguir, os limites de controle são calculados para cada um dos dois extremos do número fuzzy: $a \mathrm{e} b$.

Sejam $\tilde{x}_{11}, \ldots, \tilde{x}_{k n}$ números fuzzy triangulares para $k$ amostras com $n$ observações, representados na forma $\tilde{x}_{i j}=\left(a_{i j}, m_{i j}, b_{i j}\right)$, para $i=1, \ldots, k$ e $j=1, \ldots, n$.

Inicialmente calculam-se as médias entre os limites inferiores $\left(a_{i j}\right)$, os valores centrais $\left(m_{i j}\right)$ e os limites superiores $\left(b_{i j}\right)$ de todos os números fuzzy, conforme expressões (12), (13) e (14).

$$
\begin{aligned}
& \bar{a}=\frac{1}{k n} \sum_{i=1}^{k} \sum_{j=1}^{n} a_{i j} \\
& \bar{m}=\frac{1}{k n} \sum_{i=1}^{k} \sum_{j=1}^{n} m_{i j} \\
& \bar{b}=\frac{1}{k n} \sum_{i=1}^{k} \sum_{j=1}^{n} b_{i j}
\end{aligned}
$$

Para cada uma das $k$ amostras, determina-se a amplitude entre os valores assumidos pelos limites inferiores dos números fuzzy $\left(a_{i j}\right)$ e, posteriormente, sua média, conforme expressão (15). Procedimento similar é realizado para os imites superiores dos números fuzzy $\left(b_{i j}\right)$, conforme expressão (16).

$$
\begin{gathered}
\bar{R}_{a}=\frac{1}{k} \sum_{i=1}^{k}\left[\max \left\{a_{i 1}, \ldots, a_{i n}\right\}-\min \left\{a_{i 1}, \ldots, a_{i n}\right\}\right] \\
\bar{R}_{b}=\frac{1}{k} \sum_{i=1}^{k}\left[\max \left\{b_{i 1}, \ldots, b_{i n}\right\}-\min \left\{b_{i 1}, \ldots, b_{i n}\right\}\right]
\end{gathered}
$$

Na construção do gráfico para monitoramento da média, os limites de controle inferior $\left(L I a_{\bar{X}}\right)$ e superior $\left(L S a_{\bar{X}}\right)$ para a extremidade inferior do número fuzzy, são calculados através das expressões apresentadas em (17), enquanto que os limites de controle inferior $\left(L I_{b}\right)$ e superior $\left(L S_{b}\right)$ para a extremidade superior do número fuzzy, são calculados através das expressões apresentadas em (18): 


$$
\begin{aligned}
& \left\{\begin{array}{l}
L I a_{\bar{X}}=\bar{a}-\frac{3}{d_{2} \sqrt{n}} \bar{R}_{a} \\
L S a_{\bar{X}}=\bar{a}+\frac{3}{d_{2} \sqrt{n}} \bar{R}_{a}
\end{array}\right. \\
& \left\{\begin{array}{l}
L I b_{\bar{X}}=\bar{b}-\frac{3}{d_{2} \sqrt{n}} \bar{R}_{b} \\
L S b_{\bar{X}}=\bar{b}+\frac{3}{d_{2} \sqrt{n}} \bar{R}_{b}
\end{array}\right.
\end{aligned}
$$

Para encontrar os limites inferiores de controle fuzzy $\left(\tilde{i}_{\bar{X}}\right)$ desse gráfico, identificam-se os limites inferior $\left(\tilde{i}_{\bar{X}}\right)^{I}$ e superior $\left(\tilde{i}_{\bar{X}}\right)^{S}$ de acordo com as expressões (19).

$$
\left(\tilde{i}_{\bar{X}}\right)^{I}=\left[\min \left\{L I a_{\bar{X}}, L I b_{\bar{X}}\right\} \quad \text { e }\left(\tilde{i}_{\bar{X}}\right)^{S}=\max \left\{L I a_{\bar{X}}, L I b_{\bar{X}}\right\} .\right.
$$

De forma semelhante, para encontrar os limites superiores do gráfico de controle fuzzy $\left(\tilde{s}_{\bar{X}}\right)$ para média, identificam-se os limites inferior $\left(\tilde{s}_{\bar{X}}\right)^{I}$ e superior $\left(\tilde{S}_{\bar{X}}\right)^{S}$ de acordo com as expressões (20).

$$
\left(\tilde{s}_{\bar{X}}\right)^{I}=\left[\min \left\{L S a_{\bar{X}}, L S b_{\bar{X}}\right\} \quad \text { e } \quad\left(\tilde{s}_{\bar{X}}\right)^{S}=\max \left\{L S a_{\bar{X}}, L S b_{\bar{X}}\right\} .\right.
$$

Da mesma forma que no gráfico de controle fuzzy para a média, o processo de construção do gráfico de controle fuzzy para a amplitude se assemelha ao dos tradicionais gráficos de controle de Shewhart para a amplitude. Os limites de controle inferior $\left(L I a_{R}\right)$ e superior $\left(L S a_{R}\right)$ para o extremo inferior do número fuzzy $\left(a_{i j}\right)$ são calculados através das expressões (21), enquanto os limites de controle inferior $\left(L I b_{R}\right)$ e superior $\left(L S b_{R}\right)$ para o extremo superior do número fuzzy $\left(b_{i j}\right)$ são calculados através das expressões (22):

$$
\begin{gathered}
\left\{\begin{array}{c}
L S a_{R}=D_{4} \bar{R}_{a} \\
L I a_{R}=D_{3} \bar{R}_{a}
\end{array}\right. \\
\left\{\begin{array}{c}
L S b_{R}=D_{4} \bar{R}_{b} \\
L I b_{R}=D_{3} \bar{R}_{b}
\end{array}\right.
\end{gathered}
$$

Finalizando, para encontrar os limites inferiores de controle fuzzy $\left(\tilde{i}_{R}\right)$ deste gráfico, identificam-se os limites inferior $\left(\tilde{i}_{R}\right)^{I}$ e superior $\left(\tilde{i}_{R}\right)^{S}$ de acordo com as expressões (23). 


$$
\left(\tilde{i}_{R}\right)^{I}=\left[\min \left\{L I a_{R}, L I b_{R}\right\} \text { e }\left(\tilde{i}_{R}\right)^{S}=\max \left\{L I a_{R}, L I b_{R}\right\} . .\right.
$$

De forma semelhante, para encontrar os limites superiores do gráfico de controle fuzzy $\left(\tilde{s}_{R}\right)$ para a amplitude, identificam-se os limites inferior $\left(\tilde{s}_{R}\right)^{I}$ e superior $\left(\tilde{s}_{R}\right)^{S}$ de acordo com as expressões (24).

$$
\left(\widetilde{s}_{R}\right)^{I}=\left[\min \left\{L S a_{R}, L S b_{R}\right\} \text { e }\left(\tilde{s}_{R}\right)^{S}=\max \left\{L S a_{R}, L S b_{R}\right\} .\right.
$$

\subsubsection{Ordenação de números fuzzy}

Vários métodos já foram propostos para determinar a ordenação de números fuzzy. A escolha do método de ordenação a ser utilizado depende da forma do número fuzzy e do problema que está sendo modelado. Mais detalhes aparecem nos trabalhos de Baas e Kwakernaak (1977), Chang (1981), Bortolan e Degani (1985), Chen (1985), Kaufmann e Grupta (1988) apud Pinho et al (1997), Yuan (1991), Chen e Klein (1994), Ma e Li (2008) Shu e Wu (2011) e Asmus e Dimuro (2011).

No presente estudo será considerado o método de ordenação proposto por Yuan (1991), baseado em representação de preferências fuzzy, na racionalidade da ordenação, na distinguibilidade e na robustez. A seguir tem-se uma breve descrição do método.

Ele é utilizado para inferir se um número fuzzy $\tilde{\bar{x}}_{i}$ (ou $\tilde{r}_{i}$ para a amplitude) está entre o limite de controle superior $\tilde{s}_{\bar{X}}$ (ou $\tilde{s}_{R}$ para a amplitude) e o limite de controle inferior $\tilde{i}_{\bar{X}}$ (ou $\tilde{i}_{R}$ para a amplitude). Sejam $\tilde{N}$ e $\tilde{M}$ dois números fuzzy e $D F(\tilde{N}, \tilde{M})$ a dominância fuzzy (DF) de $\tilde{N}$ sobre $\tilde{M}$, que pode ser definida conforme expressão (25):

$$
\operatorname{DF}(\tilde{N}, \tilde{M})=\frac{\Delta_{\tilde{N}, \tilde{M}}}{\Delta_{\tilde{N}, \tilde{M}}+\Delta_{\tilde{M}, \tilde{N}}}
$$

onde:

$$
\begin{aligned}
& \Delta_{\tilde{N}, \tilde{M}}=\int_{\left\{\alpha: \tilde{N}_{\alpha}^{S}>\tilde{M}_{\alpha}^{I}\right\}}\left(\tilde{N}_{\alpha}^{S}-\tilde{M}_{\alpha}^{I}\right) d \alpha+\int_{\left\{\alpha: \tilde{N}_{\alpha}^{I}>\tilde{M}_{\alpha}^{S}\right\}}\left(\tilde{N}_{\alpha}^{I}-\tilde{M}_{\alpha}^{S}\right) d \alpha \\
& \Delta_{\tilde{M}, \tilde{N}}=\int_{\left\{\alpha: \tilde{M}_{\alpha}^{S}>\tilde{N}_{\alpha}^{I}\right\}}\left(\tilde{M}_{\alpha}^{S}-\tilde{N}_{\alpha}^{I}\right) d \alpha+\int_{\left\{\alpha: \tilde{M}_{\alpha}^{I}>\tilde{N}_{\alpha}^{S}\right\}}\left(\tilde{M}_{\alpha}^{I}-\tilde{N}_{\alpha}^{S}\right) d \alpha
\end{aligned}
$$


$\mathrm{Na}$ expressão (26), a integral apresentada na primeira parcela da soma significa a região onde $\tilde{N}^{S}$ domina $\tilde{M}^{I}$, enquanto que a apresentada na segunda parcela dessa soma é a região onde $\tilde{N}^{I}$ domina $\tilde{M}^{S}$. Logo, $\Delta_{\tilde{N}, \tilde{M}}$ significa o somatório das regiões onde $\tilde{N}$ domina $\tilde{M}$. Na expressão (27) tem-se o somatório das regiões onde $\tilde{M}$ domina $\tilde{N}$.

Shu e Wu (2011) complementam esse método propondo a adoção de um nível de dominância $\gamma$ que pertence ao intervalo $[0,5 ; 1,0]$ e pode variar conforme o grau de rigor necessário. As regras de avaliação propostas pelo autor são:

(a) se $D F(\tilde{M}, \tilde{N})>\gamma$, então se escreve $\tilde{M} \succ_{\gamma} \tilde{N}$. De forma equivalente, se $D F(\tilde{N}, \tilde{M})<1-\gamma$ então $\tilde{N} \succ_{\gamma} \tilde{M}$. Nesse caso tem-se que $\tilde{M}$ é maior do que $\tilde{N}$ com $\gamma$ graus de dominância, ou $\tilde{N}$ é menor do que $\tilde{M}$ com $\gamma$ graus de dominância;

(b) se $D F(\tilde{M}, \tilde{N})<1-\gamma$, conforme (a), tem-se que $D F(\tilde{N}, \tilde{M})>\gamma$, ou seja, $\tilde{N} \succ_{\gamma} \tilde{M}$, o que significa que $\tilde{N}$ é maior do que $\tilde{M}$ com $\gamma$ graus de dominância;

(c) se $0,5 \leq D F(\tilde{M}, \tilde{N}) \leq \gamma$, então se escreve $\tilde{M} \approx_{\gamma} \tilde{N}$, que é equivalente a $1-\gamma \leq D F(\tilde{N}, \tilde{M}) \leq 0,5$ de acordo com (a). Nesse caso tem-se que $\tilde{M}$ é fortemente indiferente de $\tilde{N}$ com $\gamma$ graus de dominância, indicando que $\tilde{M}$ não domina $\tilde{N}$ de forma significativa;

(d) se $1-\gamma \leq D F(\tilde{M}, \tilde{N}) \leq 0,5$, então se escreve que $\tilde{M} \dot{ }_{\gamma} \tilde{N}$. De forma equivalente, conforme (a), $0,5 \leq D F(\tilde{N}, \tilde{M}) \leq \gamma$. Nesse caso, tem-se que $\tilde{M}$ é fracamente indiferente de $\tilde{N}$ com $\gamma$ graus de dominância. Essa diferença indica que $\tilde{M}$ é fracamente dominado por $\tilde{N}$.

Com essas regras de avaliação é usual categorizar o processo de produção considerando $\gamma=0,5$ e um outro valor superior a este $(\gamma>0,5)$, separadamente.

\section{ESTUDO DE CASO}

\subsection{Contextualização}


O estudo foi desenvolvido em um processo produtivo de uma bebida fermentada, o iogurte, sendo as observações realizadas na linha de produção do iogurte do sabor pêssego. Na identificação das características definidoras de sua qualidade foram selecionadas algumas características sensoriais, difíceis de serem mensuradas por depender da percepção dos indivíduos. São elas: cor, aroma, consistência, sabor e acidez.

Para avaliá-las, cinco juízes, individualmente, atribuíram uma nota a cada uma dessas características sensoriais de amostras do alimento avaliado, localizando um ponto em um segmento de reta que possui suas extremidades relacionadas a zero (péssimo) e dez (excelente). Essa avaliação foi realizada junto ao processo produtivo ao longo de dez semanas. Em cada semana foram feitas duas coletas e em cada coleta foram extraídas quatro unidades amostrais. Um feriado nesse período fez com que fossem realizadas 19 coletas em lugar das 20 esperadas.

Para cada característica sensorial e cada unidade amostral do produto, as cinco informações fornecidas pelos juízes foram substituídas pela média aritmética, sendo essa utilizada para a construção dos tradicionais gráficos de controle Shewhart para a média e para a amplitude.

Estas mesmas observações também foram utilizadas para gerar um número fuzzy triangular, utilizado na construção dos gráficos de controle fuzzy, o que foi feito por meio de uma adaptação dos gráficos propostos em Shu e Wu (2011), descrita resumidamente na seção anterior.

Para tal, inicialmente as avaliações dos juízes foram transformadas em números fuzzy pelo método proposto em Cheng (2005). A interpretação dos gráficos de controle fuzzy foi feita por meio de uma avaliação da dominância em relação aos limites de controle pelo método proposto por Yuan (1991) e expandido por Shu e Wu (2011), também descritos na seção anterior. Nesta interpretação foram considerados dois níveis de dominância $(\gamma)$ : 0,5 e 0,7. A definição de utilização de cinco observações para construir os números fuzzy se embasou em estudo desenvolvido por Bartlett e Kendall (1946) que mostrou a necessidade de utilização de pelo menos cinco replicações em experimentos para obtenção de estimação eficiente da variabilidade, mais especificamente, a variância, já que a técnica trabalha com a variância das possibilidades. Finalizando, as interpretações obtidas com os cinco gráficos de controle de Shewhart para análise da média das características 
sensoriais foram comparadas com as interpretações obtidas com os cinco gráficos de controle fuzzy para esta mesma medida e características, o mesmo acontecendo com os dez gráficos de controle construídos para a amplitude (Shewhart e fuzzy).

No presente artigo são apresentados alguns resultados parciais, incluindo os gráficos de controle, apenas para a característica "cor", sendo os resultados das demais características apresentados apenas na comparação das interpretações dos gráficos.

\subsection{Resultados}

Inicialmente foi desenvolvida uma análise exploratória nas observações brutas, utilizando técnicas gráficas e analíticas, com o objetivo de conhecer algumas de suas propriedades, que divergiram em termos de tendência central e variabilidade, conforme mostrado na Figura 1. Também fica evidenciada a baixa qualidade do produto final, principalmente em relação à característica cor.

Figura 1 - Box Plot construídos com as mensurações das características sensoriais

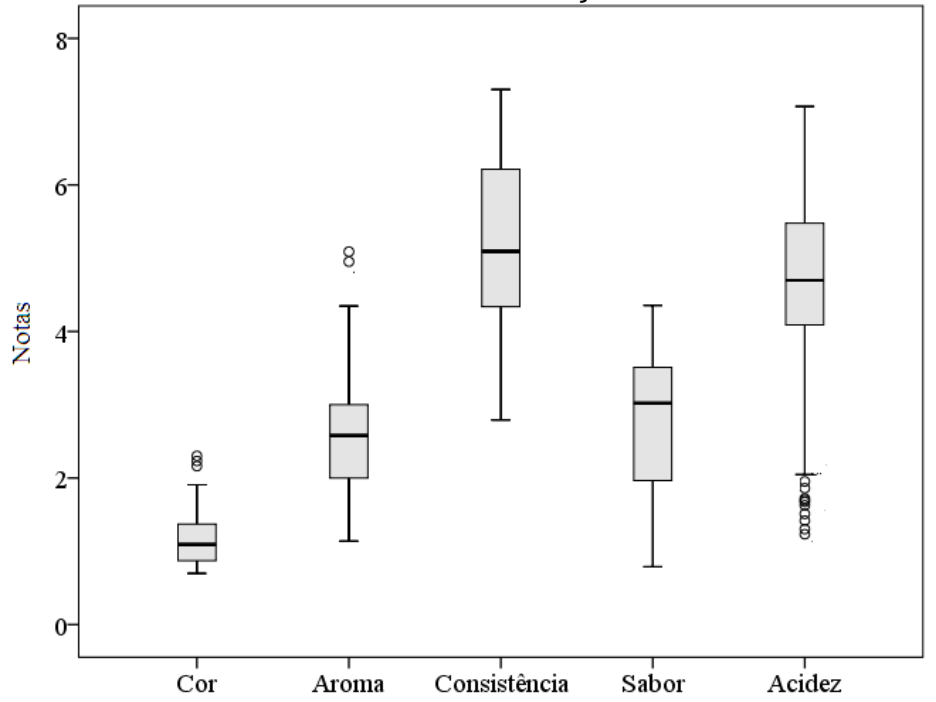

Fonte: os autores

Para cada característica sensorial foi gerado um conjunto de observações constituído por 76 médias (que substituem as notas dos cinco juízes) que foram utilizadas para construir os gráficos de controle de Shewhart (média e amplitude), 0 primeiro item da primeira amostra analisada recebeu para a característica cor as pontuações: $g_{111}=0,555556 ; \quad g_{112}=1,111111 ; \quad g_{113}=1,222222 ; \quad g_{114}=1,666667$ e $g_{115}=1,888889$, fornecendo a média $\bar{g}_{11}=1,2888889$. 
A Figura 2 apresenta o gráfico de controle de Shewhart para a média, que aponta fortes indícios de instabilidade no processo, além de indicar que a característica em questão foi muito mal avaliada, pois a partir da amostra 6 observam-se valores abaixo do limite inferior de controle $(6,7,9,10,11$ e 12). Ainda, existem cinco pontos acima do limite superior de controle $(3,13,15,17$ e 18), totalizando 11 pontos fora dos limites de controle. Entre a extração da amostra $14 \mathrm{e}$ a extração da amostra 15 houve uma causa especial, cujo efeito foi detectado pelo referido gráfico: aumento dos resultados médios. O gráfico sugere, entretanto, que isso tenha ocorrido a partir da amostra 12.

Figura 2 - Gráfico de controle de Shewhart para a média da característica cor

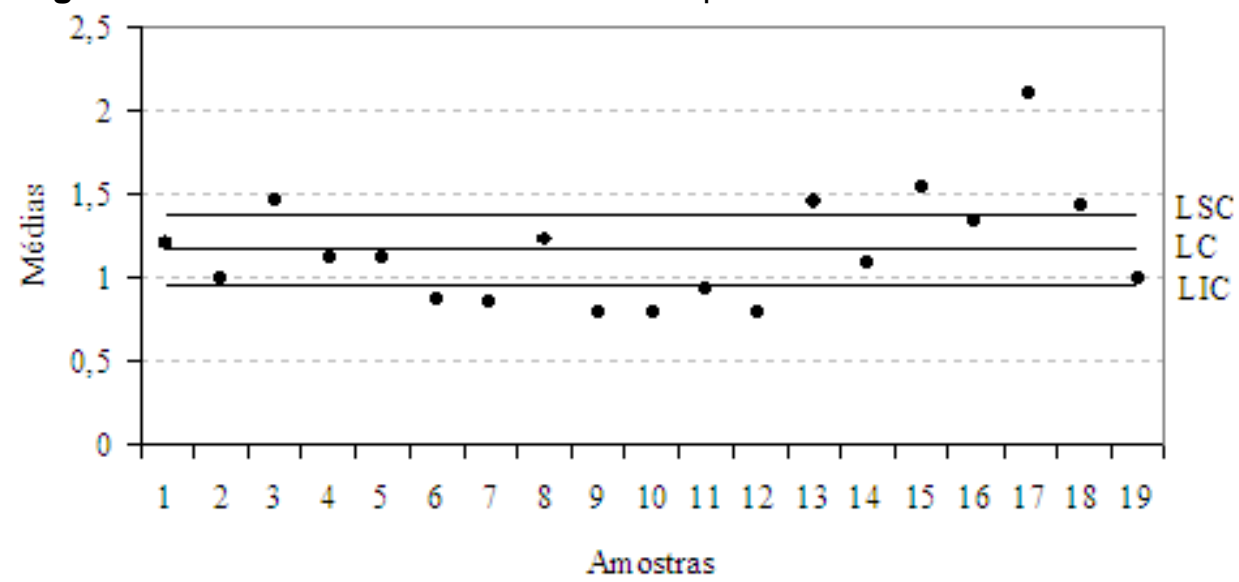

Fonte: os autores

OBS: LIC (Limite Inferior de Controle) $=0,9554$; LC (Linha Central) $=1,1688$; LSC (Limite Superior de Controle) $=1,3822$.

A Figura 3 apresenta o gráfico de controle de Shewhart para a amplitude que não mostra nenhuma evidência aparente de instabilidade por não existirem pontos fora dos limites de controle. Portanto, o processo pode ser considerado estável quanto à variabilidade na cor do iogurte. Observa-se, entretanto, que a partir da amostra 13 existem duas amostras com grande variabilidade e cinco amostras em torno da linha central, sugerindo que pode ter havido um aumento na amplitude amostral. Importante ficar atento. 
Figura 3 - Gráfico de controle de Shewhart para a amplitude da característica cor

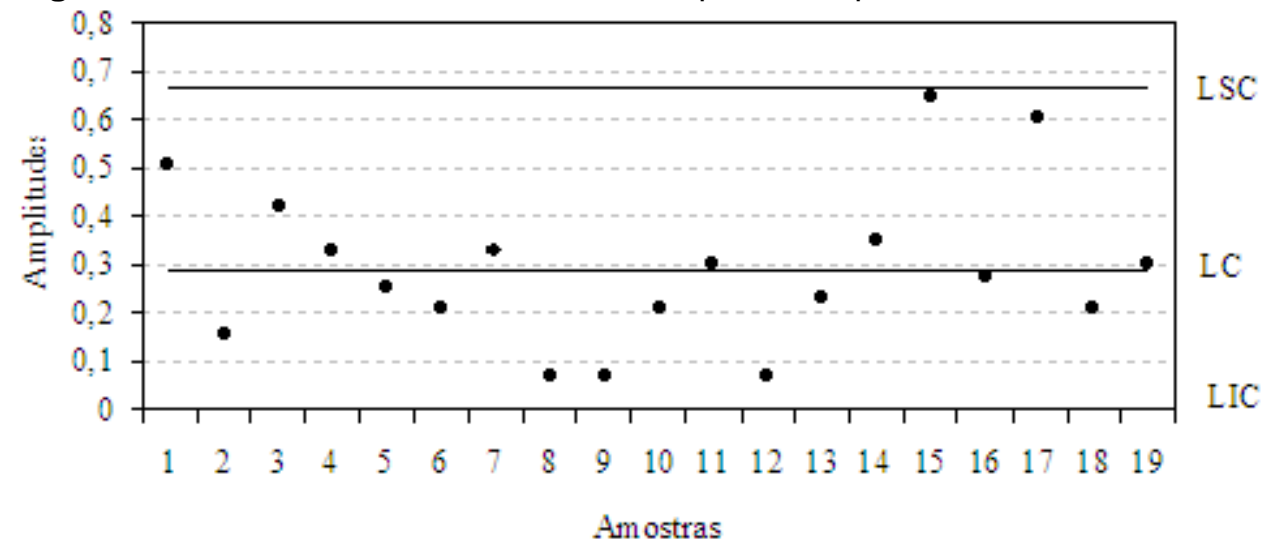

Fonte: os autores.

OBS: LIC (Limite Inferior de Controle) = 0,0000; LC (Linha Central) = 0,2927; LSC (Limite Superior de Controle) $=0,6679$.

Para cada característica sensorial também foi gerado um conjunto de observações constituído por 76 números fuzzy (que substituem as notas dos cinco juízes) que foram usadas na construção dos gráficos de controle fuzzy (média e amplitude).

O número fuzzy correspondente ao primeiro item da primeira amostra analisada, $\tilde{x}_{11}=\left(a_{11}, m_{11}, b_{11}\right)$, apresentado anteriormente, foi encontrado conforme 0 descrito a seguir. Inicialmente foi determinada a matriz D, conforme expressão (1), fornecendo subsídios para a determinação das distâncias médias (expressão 2). Foram encontrados os valores: $\bar{d}_{1}=0,916663 ; \quad \bar{d}_{2}=0,500000 ; \bar{d}_{3}=0,472223$; $\bar{d}_{4}=0,583335 \mathrm{e} \cdot \bar{d}_{5}=0,750000$.

$$
D=\left[\begin{array}{ccccc}
0,00000 & 0,55555 & 0,66666 & 1,11111 & 1,33333 \\
0,55555 & 0,00000 & 0,11111 & 0,55556 & 0,77778 \\
0,666667 & 0,11111 & 0,00000 & 0,44445 & 0,66667 \\
1,11111 & 0,55556 & 0,44445 & 0,00000 & 0,22222 \\
1,33333 & 0,77778 & 0,66667 & 0,22222 & 0,00000
\end{array}\right]
$$

O grau de importância de cada nota, comparada às demais, fornece valores para $p_{i j}$, conforme uma das expressões (3), organizados na matriz $P$.

$$
P=\left[\begin{array}{lllll}
1,00000 & 0,54546 & 0,51515 & 0,63637 & 0,81819 \\
1,83333 & 1,00000 & 0,94445 & 1,16667 & 1,50000 \\
1,94117 & 1,05882 & 1,00000 & 1,23530 & 1,58823 \\
1,57142 & 0,85714 & 0,80952 & 1,00000 & 1,28571 \\
1,22222 & 0,66667 & 0,62963 & 0,77778 & 1,00000
\end{array}\right]
$$


A partir destas informações, são determinados pela outra expressão (3) os pesos $w_{i}$, sendo encontrados os seguintes resultados: $w_{1}=0,132133 ; w_{2}=0,242243$; $w_{3}=0,256492 ; w_{4}=0,207636$ e $w_{5}=0,161495$. A expressão (4) permite encontrar o valor central do número fuzzy: $m=1,307165$.

Para estimar a variância das possibilidades é usada a expressão (5), sendo encontrado $\hat{S}^{2}=0,178382$. As expressões (6) e (7) fornecem os resultados de $g^{l}$ e $g^{r}$, que são respectivamente, 1,039926 e 1,763891. A expressão (8) fornece uma estimativa da razão da distribuição dos dados em torno do valor central: $\hat{\rho}=0,585116$. Finalizando, os extremos do número fuzzy são calculados usando as expressões (9) e (10), sendo encontrados os valores $a=0,568286$ e $b=2,569954$. O número fuzzy determinado é $\tilde{x}_{11}=\left(a_{11}, m_{11}, b_{11}\right)=(0,5683 ; 1,3072 ; 2,5700)$.

Para a construção dos gráficos de controle fuzzy são calculados os valores médios, conforme o definido pelas expressões (12), (13), (14), (15) e (16), obtendose para a variável cor os resultados: $\bar{a}=-0,32427 ; \bar{b}=2,909665 ; \bar{R}_{a}=0,684149$ e $\bar{R}_{b}=1,268239$.

Estes valores são utilizados no cálculo dos limites de controle. No gráfico fuzzy para a média da variável cor, os limites de controle inferior $\left(L I a_{\bar{X}}=-0,82268\right)$ e superior $\left(L S a_{\bar{X}}=0,174142\right)$ para a extremidade inferior do número fuzzy são determinados pelas expressões (17), enquanto os limites de controle inferior $\left(L I_{b}=\right.$ 1,985743) e superior ( $L S_{b}=3,833588$ ) para a extremidade superior do número fuzzy,são calculados pelas expressões (18).

Finalizando, o limite inferior de controle fuzzy $\left(\tilde{i}_{\bar{X}}\right)$, determinado pelas expressões (19) e o limite superior do gráfico de controle fuzzy $\left(\tilde{s}_{\bar{X}}\right)$, determinado pelas expressões (20), fornecem os seguintes resultados para a variável cor: $\tilde{i}_{\bar{X}}=\{-0,82268 ; 1,985743\}$ e $\tilde{s}_{\bar{X}}=\{0,174142 ; 3,833588\}$.

A Figura 4 apresenta o gráfico de controle fuzzy para a média, que também encontra evidencias de instabilidade, pois as amostras 3, 13 e 17 parecem dominar o limite de controle superior. Essas evidências são comprovadas pelas informações apresentadas na Tabela 1, que mostra os resultados encontrados por meio da expressão 25 para dois níveis de dominância e respectiva interpretação.

Revista Produção Online, Florianópolis, SC, v. 16, n. 2, p. 754-778, abr./jun. 2016. 
Nesse caso, considerando $\gamma=0,5$, as regras apresentadas na seção anterior, reduzem-se a: (a) se $D F\left(\tilde{s}_{\bar{X}}, \tilde{\bar{x}}_{i}\right)>0,5$ e $D F\left(\tilde{\bar{x}}_{i}, \tilde{i}_{\bar{X}}\right)>0,5$, o processo estará em controle; (b) se $D F\left(\tilde{s}_{\bar{X}}, \tilde{\bar{x}}_{i}\right)<0,5$ ou $D F\left(\tilde{\bar{x}}_{i}, \tilde{i}_{\bar{X}}\right)<0,5$, o processo estará fora de controle; (c) estará um pouco em controle se $D F\left(\tilde{s}_{\bar{X}}, \tilde{\bar{x}}_{i}\right)=0,5$ e $D F\left(\tilde{\bar{x}}_{i}, \tilde{i}_{\bar{X}}\right)>0,5$ ou $D F\left(\tilde{s}_{\bar{X}}, \tilde{\bar{x}}_{i}\right)>0,5$ e $D F\left(\tilde{\bar{x}}_{i}, \tilde{i}_{\bar{X}}\right)=0,5$.

Considerando $\gamma=0,7$, as regras apresentadas na seção anterior, reduzem-se a: (a) se $D F\left(\widetilde{s}_{\bar{X}}, \tilde{\bar{x}}_{i}\right)>0,7$ e $D F\left(\tilde{\bar{x}}_{i}, \tilde{i}_{\bar{X}}\right)>0,7$, o processo estará em controle; (b) se $D F\left(\tilde{s}_{\bar{X}}, \tilde{\bar{x}}_{i}\right)<0,3$ e $D F\left(\tilde{\bar{x}}_{i}, \tilde{\bar{X}}_{\bar{X}}\right)<0,3$, o processo estará fora de controle; (c) se $0,3<D F\left(\tilde{s}_{\bar{X}}, \tilde{\bar{x}}_{i}\right)<0,5$ ou $0,3<D F\left(\tilde{\bar{x}}_{i}, \tilde{i}_{\bar{X}}\right)<0,5$, então o processo estará um pouco fora de controle; (d) o processo estará um pouco em controle se (d.1) $D F\left(\tilde{s}_{\bar{X}}, \tilde{\bar{x}}_{i}\right)>0,7$ e $0,5 \leq D F\left(\tilde{\bar{x}}_{i}, \tilde{i}_{\bar{X}}\right) \leq 0,7$, (d.2) $D F\left(\tilde{\bar{x}}_{i}, \tilde{i}_{\bar{X}}\right)>0,7$ e $0,5 \leq D F\left(\tilde{s}_{\bar{X}}, \tilde{\bar{x}}_{i}\right) \leq 0,7$ ou (d.3) $0,5 \leq D F\left(\tilde{s}_{\bar{X}}, \tilde{\bar{x}}_{i}\right) \leq 0,7$ e $0,5 \leq D F\left(\tilde{\bar{x}}_{i}, \tilde{i}_{\bar{X}}\right) \leq 0,7$

Para $\gamma=0,5$, as amostras 3, 13 e 17 são avaliadas como fora de controle, enquanto as demais, sob controle. Para um nível de dominância mais elevado $(\gamma=0,7)$, nenhuma das amostras é considerada absolutamente sob controle. As amostras 3, 13 e 17 são consideradas pouco fora de controle, enquanto as demais, pouco em controle. Observa-se que as interpretações são similares para os dois níveis de dominância na interpretação, pois foram formados dois grupos. Para menor rigor $(\gamma=0,5)$, a interpretação das observações fica mais diferente (em controle ou fora de controle), o mesmo não acontecendo para um rigor de interpretação maior (pouco em controle ou pouco fora de controle).

Observe que, ao aumentar o rigor, criam-se zonas de alerta (pouco em controle e pouco fora de controle). Nos tradicionais gráficos de controle de Shewhart também podem ser criadas zonas de alerta com a consideração de linhas situadas a um e dois desvios padrão da média. Essas zonas valem, entretanto, apenas para valores que ficam dentro dos limites de controle, o mesmo não acontecendo com os gráficos de controle fuzzy. Observe, entretanto, que a causa especial que ocorreu entre a extração da amostra 14 e a extração da amostra 15, não foi claramente detectada por este gráfico, embora as amplitudes dos números fuzzy que 
representam as amostras (Figura 5), de maneira geral, aumentaram a partir da amostra 13.

Figura 4 - Gráfico de controle fuzzy para a média da característica cor

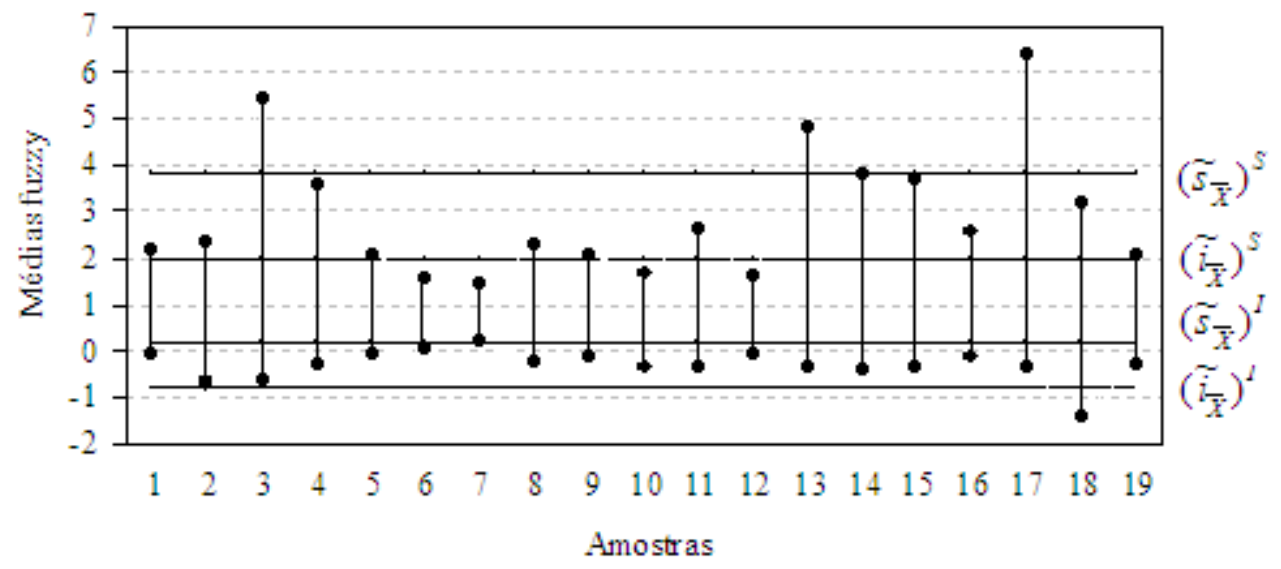

Fonte: os autores

OBS: $\left(\tilde{s}_{\bar{X}}\right)^{S}$ (Limite superior de controle do limite superior do número fuzzy) $=3,833588$

$\left(\tilde{i}_{\bar{X}}\right)^{S}$ (Limite inferior de controle do limite superior do número fuzzy) $=1,985743$

$\left(\tilde{s}_{\bar{X}}\right)^{I}$ (Limite superior de controle do limite inferior do número fuzzy) $=0,174142$

$\left(\tilde{i}_{\bar{X}}\right)^{I}$ (Limite e inferior de controle do limite inferior do número fuzzy) $=-0,82268$

Tabela 1 - Avaliação do gráfico de controle fuzzy para a média da característica cor, considerando $\gamma=0,5$ e $\gamma=0,7$

\begin{tabular}{ccccc}
\hline Amostras & $D F\left(\tilde{s}_{\bar{X}}, \tilde{\bar{x}}_{i}\right)$ & $D F\left(\tilde{\bar{x}}_{i}, \tilde{i}_{\bar{X}}\right)$ & $\begin{array}{c}\text { Processo para } \\
\gamma=0,5\end{array}$ & $\begin{array}{c}\text { Processo para } \\
\gamma=0,7\end{array}$ \\
\hline 1 & 0,6960 & 0,6081 & Em Controle & Pouco em Controle \\
2 & 0,7204 & 0,5226 & Em Controle & Pouco em Controle \\
3 & 0,4507 & 0,7074 & Fora de Controle & Pouco Fora de Controle \\
4 & 0,5602 & 0,6414 & Em Controle & Pouco em Controle \\
5 & 0,7181 & 0,5788 & Em Controle & Pouco em Controle \\
6 & 0,8035 & 0,5543 & Em Controle & Pouco em Controle \\
7 & 0,8243 & 0,5638 & Em Controle & Pouco em Controle \\
8 & 0,7047 & 0,5734 & Em Controle & Pouco em Controle \\
9 & 0,7395 & 0,5110 & Em Controle & Pouco em Controle \\
10 & 0,8131 & 0,5161 & Em Controle & Pouco em Controle \\
11 & 0,6761 & 0,5434 & Em Controle & Pouco em Controle \\
12 & 0,8075 & 0,5428 & Em Controle & Pouco em Controle \\
13 & 0,4713 & 0,7180 & Fora de Controle & Pouco Fora de Controle \\
14 & 0,5474 & 0,6253 & Em Controle & Pouco em Controle \\
15 & 0,5482 & 0,6951 & Em Controle & Pouco em Controle \\
16 & 0,6514 & 0,6420 & Em Controle & Pouco em Controle \\
17 & 0,4016 & 0,7941 & Fora de Controle & Pouco Fora de Controle \\
18 & 0,6554 & 0,5533 & Em Controle & Pouco em Controle \\
19 & 0,7338 & 0,5407 & Em Controle & Pouco em Controle \\
\hline
\end{tabular}

Fonte: os autores

Revista Produção Online, Florianópolis, SC, v. 16, n. 2, p. 754-778, abr./jun. 2016. 
Observe ainda que as amostras 3, 13 e 17, detectadas como evidência de situação fora de controle em ambos os gráficos, indicam um aumento no resultado, segundo avaliação dos juizes. Entretanto, as amostras que sugerem diminuição do resultado no gráfico de Shewhart não foram detectadas pelo gráfico fuzzy. Esses achados sugerem que os tradicionais gráficos de controle de Shewhart podem ter o inconveniente de apresentar uma quantidade maior de possíveis falsos alarmes, concordando com Joekes, Smrekar e Barbosa (2015), quando comparados com a adaptação dos gráficos fuzzy propostos por Shu e Wu (2011). Salienta-se, entretanto, que Joekes, Smrekar e Barbosa (2015) analisaram um processo de alta qualidade, bastante diferente do apresentado neste estudo.

Para a construção do gráfico de controle fuzzy para a amplitude da variável cor, os limites de controle inferior e superior para o extremo inferior do número fuzzy, determinados pelas expressões (21), fornecem os resultados $L I a_{R}=0,0$ e $L S a_{R}=$ 1,561229, respectivamente. Para os limites de controle inferior e superior do extremo superior do número fuzzy, determinados pela expressões (22), são encontrados os valores $L I b_{R}=0,0$ e $L S b_{R}=2,894119$, respectivamente. Seu limite inferior de controle fuzzy $\left(\tilde{i}_{\bar{X}}\right)$, determinado pelas expressões (23) e seu limite superior de controle fuzzy $\left(\tilde{s}_{\bar{X}}\right)$, determinado pelas expressões (24), fornecem os seguintes resultados para a variável cor: $\tilde{i}_{R}=\{0 ; 0\}$ e $\tilde{s}_{R}=\{1,561229 ; 2,894119\}$. As regras usadas em sua interpretação são as mesmas utilizadas na interpretação do gráfico de controle fuzzy para a média.

A Figura 5 apresenta o gráfico de controle fuzzy para a amplitude, sendo visualmente detectada uma amostra fora dos limites de controle (amostra 15). Ao aplicar as regras de interpretação para os resultados de dominância mostrados na Tabela 2, observa-se que, considerando um nível de dominância menor $(\gamma=0,5)$, não são encontradas evidências de instabilidade, pois todas as amostras são consideradas em controle. Quando o rigor na interpretação aumenta $(\gamma=0,7)$, a amostra 15 é considerada pouco em controle, concordando com a interpretação feita pela aparência visual do gráfico. Nesse caso, o gráfico de controle fuzzy com nível de dominância maior foi mais sensível. 
Figura 5 - Gráfico de controle fuzzy para a amplitude da característica cor

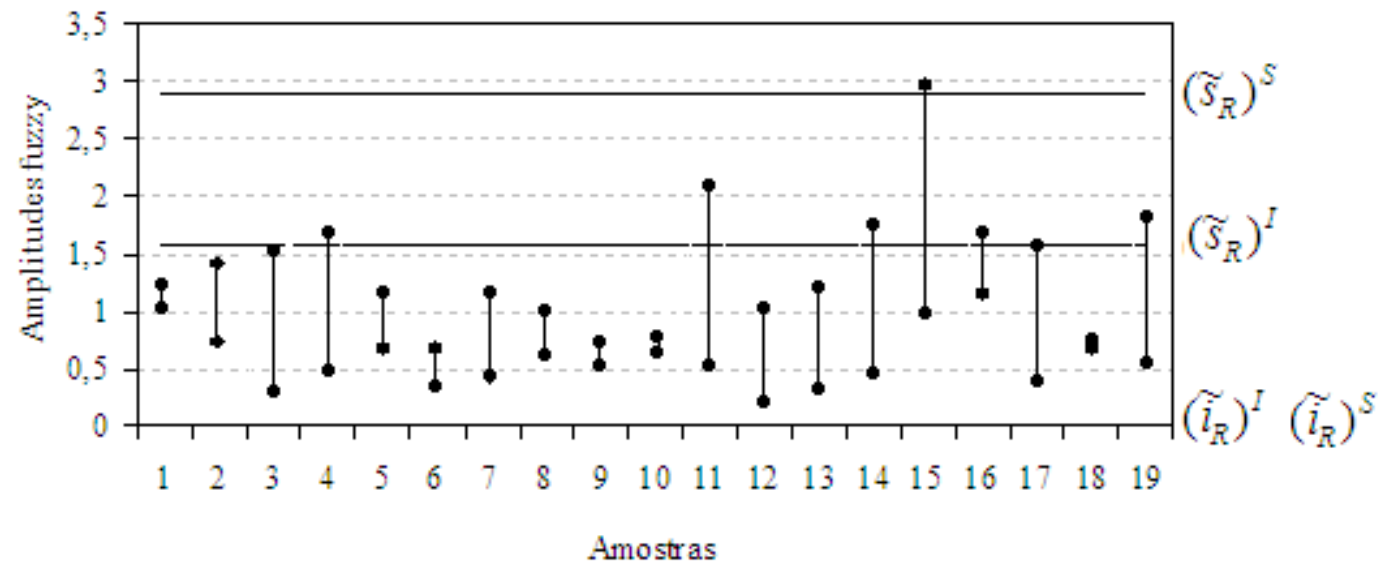

Fonte: os autores

OBS: $\left(\tilde{s}_{R}\right)^{S}$ (Limite superior de controle do limite superior do número fuzzy) $=2,894119$

$\left(\tilde{i}_{R}\right)^{S}$ (Limite inferior de controle do limite superior do número fuzzy) $=0,00$

$\left(\widetilde{s}_{R}\right)^{I}$ (Limite superior de controle do limite inferior do número fuzzy) $=1,561229$

$\left(\tilde{i}_{R}\right)^{I}$ (Limite inferior de controle do limite inferior do número fuzzy) $=0,00$

Tabela 2 - Avaliação do gráfico de controle fuzzy para a amplitude da característica cor, considerando $\gamma=0,5$ e $\gamma=0,7$

\begin{tabular}{ccccc}
\hline Amostras & $D F\left(\tilde{s}_{\bar{X}}, \tilde{\bar{x}}_{i}\right)$ & $D F\left(\tilde{\bar{x}}_{i}, \tilde{i}_{\bar{X}}\right)$ & $\begin{array}{c}\text { Processo para } \\
\gamma=0,5\end{array}$ & $\begin{array}{c}\text { Processo para } \\
\gamma=0,7\end{array}$ \\
\hline 1 & 1,0000 & 1,0000 & Em Controle & Em Controle \\
2 & 1,0000 & 1,0000 & Em Controle & Em Controle \\
3 & 1,0000 & 1,0000 & Em Controle & Em Controle \\
4 & 0,9951 & 1,0000 & Em Controle & Em Controle \\
5 & 1,0000 & 1,0000 & Em Controle & Em Controle \\
6 & 1,0000 & 1,0000 & Em Controle & Em Controle \\
7 & 1,0000 & 1,0000 & Em Controle & Em Controle \\
8 & 1,0000 & 1,0000 & Em Controle & Em Controle \\
9 & 1,0000 & 1,0000 & Em Controle & Em Controle \\
10 & 1,0000 & 1,0000 & Em Controle & Em Controle \\
11 & 0,9163 & 1,0000 & Em Controle & Em Controle \\
12 & 1,0000 & 1,0000 & Em Controle & Em Controle \\
13 & 1,0000 & 1,0000 & Em Controle & Em Controle \\
14 & 0,9890 & 1,0000 & Em Controle & Em Controle \\
15 & 0,5906 & 1,0000 & Em Controle & Pouco em Controle \\
16 & 0,9914 & 1,0000 & Em Controle & Em Controle \\
17 & 1,0000 & 1,0000 & Em Controle & Em Controle \\
18 & 1,0000 & 1,0000 & Em Controle & Em Controle \\
19 & 0,9780 & 1,0000 & Em Controle & Em Controle \\
\hline
\end{tabular}

Fonte: os autores

Portanto, para a característica de qualidade sensorial cor, o tradicional gráfico de Shewhart para a média aponta evidências de instabilidade para a tendência Revista Produção Online, Florianópolis, SC, v. 16, n. 2, p. 754-778, abr.jjun. 2016. 
central, o mesmo não acontecendo com o gráfico de controle para a amplitude. $\mathrm{O}$ gráfico de controle fuzzy para a média, no nível de dominância $\gamma=0,5$, captou instabilidade no processo, considerando três amostras fora de controle. As mesmas amostras são consideradas pouco fora de controle para $\gamma=0,7$. No gráfico de controle fuzzy para a amplitude, para o grau de dominância $\gamma=0,7$, a amostra 15 foi considerada pouco em controle, enquanto que para $\gamma=0,5$, todas estão sob controle.

Esses achados sugerem que o gráfico de controle de Shewhart para a média consegue identificar a ocorrência da causa especial, o que não ficou tão evidente no gráfico de controle fuzzy. Para a amplitude, entretanto, o gráfico de controle fuzzy apresentou maior sensibilidade.

Análise similar foi desenvolvida para as demais características sensoriais (aroma, consistência, sabor e acidez) e o resumo da interpretação dos resultados encontrados para a tendência central estão apresentados no Quadro 1, enquanto o resumo dos resultados encontrados para a amplitude estão apresentados no Quadro 2.

Pelas informações do Quadro 1, é possível constatar que os tradicionais gráficos de controle de Shewhart foram mais rigorosos que o gráficos de controle fuzzy na análise das informações, identificando uma quantidade maior de pontos fora dos limites de controle. Nos gráficos de controle fuzzy, a utilização de $\gamma=0,5$ identifica menos evidências de pontos fora dos limites de controle, mas, com o aumento do nível de dominância para $\gamma=0,7$, são identificados mais pontos pouco em controle. Nenhum, entretanto, fora de controle. 
Quadro 1 - Resumo da interpretação dos resultados dos gráficos de controle para média

\begin{tabular}{|c|c|c|c|}
\hline \multirow[t]{2}{*}{ CARACTERISTICA } & \multirow[t]{2}{*}{ SHEWHART } & \multicolumn{2}{|c|}{ FUZZY } \\
\hline & & $\gamma=0,5$ & $\gamma=0,7$ \\
\hline Cor & $\begin{array}{l}11 \text { pontos fora dos } \\
\text { limites de controle }\end{array}$ & $\begin{array}{l}3 \text { pontos fora dos limites } \\
\text { de controle }\end{array}$ & $\begin{array}{l}3 \text { pontos pouco fora de } \\
\text { controle } \\
16 \text { pontos pouco em } \\
\text { controle }\end{array}$ \\
\hline Aroma & $\begin{array}{l}5 \text { pontos fora dos } \\
\text { limites de controle }\end{array}$ & $\begin{array}{l}3 \text { pontos fora dos limites } \\
\text { de controle }\end{array}$ & $\begin{array}{l}3 \text { pontos pouco fora de } \\
\text { controle } \\
16 \text { pontos pouco em } \\
\text { controle }\end{array}$ \\
\hline Consistência & $\begin{array}{l}12 \text { pontos fora dos } \\
\text { limites de controle }\end{array}$ & Em controle & $\begin{array}{l}19 \text { pontos pouco em } \\
\text { controle }\end{array}$ \\
\hline Sabor & $\begin{array}{l}9 \text { pontos fora dos } \\
\text { limites de controle }\end{array}$ & $\begin{array}{l}2 \text { pontos fora dos limites } \\
\text { de controle }\end{array}$ & $\begin{array}{l}2 \text { pontos pouco fora de } \\
\text { controle } \\
17 \text { pontos pouco em } \\
\text { controle }\end{array}$ \\
\hline Acidez & $\begin{array}{l}6 \text { pontos fora dos } \\
\text { limites de controle }\end{array}$ & $\begin{array}{l}1 \text { ponto fora dos limites } \\
\text { de controle }\end{array}$ & $\begin{array}{l}1 \text { ponto pouco fora de } \\
\text { controle } \\
18 \text { pontos pouco em } \\
\text { controle }\end{array}$ \\
\hline
\end{tabular}

Fonte: os autores

Pelas informações do Quadro 2, observa-se que os gráficos tradicionais de Shewhart para amplitude identificam apenas um ponto fora dos limites de controle para uma das características, enquanto que os gráficos de controle fuzzy, com a utilização de nível de dominância $\gamma=0,5$, consideram todas as características sob controle estatístico. Já a interpretação do gráfico de controle fuzzy com a utilização de nível de dominância $\gamma=0,7$ parece ser a mais sensível, identificando um ponto pouco em controle para três características. É possível que isso ocorra pelo fato de que os números fuzzy levam em consideração a variabilidade existente entre as avaliações dos juízes sobre uma mesma unidade amostral, o mesmo não acontecendo com as informações usadas nos gráficos de controle de Shewhart que consideram apenas a sua média.

Quadro 2 - Resumo da interpretação dos resultados dos gráficos de controle para amplitude

\begin{tabular}{|c|c|c|c|}
\hline \multirow[t]{2}{*}{ CARACTERÍSTICA } & \multirow[t]{2}{*}{ SHEWHART } & \multicolumn{2}{|c|}{ FUZZY } \\
\hline & & $\gamma=0,5$ & $\gamma=0,7$ \\
\hline Cor & Sob controle & Sob controle & $\begin{array}{l}1 \text { ponto pouco em } \\
\text { controle }\end{array}$ \\
\hline Aroma & Sob controle & Sob controle & $\begin{array}{l}1 \text { ponto pouco em } \\
\text { controle }\end{array}$ \\
\hline Consistência & Sob controle & Sob controle & Sob controle \\
\hline Sabor & Sob controle & Sob controle & $\begin{array}{l}1 \text { ponto pouco em } \\
\text { controle }\end{array}$ \\
\hline Acidez & $\begin{array}{l}1 \text { ponto fora dos } \\
\text { limites de controle }\end{array}$ & Sob controle & Sob controle \\
\hline
\end{tabular}

Fonte: os autores

Revista Produção Online, Florianópolis, SC, v. 16, n. 2, p. 754-778, abr./jun. 2016. 


\section{CONSIDERAÇÕES FINAIS}

As empresas necessitam de produtos que sejam industrialmente rentáveis e atendam às exigências dos consumidores, razão pela qual, estudar e aprimorar técnicas que possam melhorar a qualidade de processos produtivos pode ser extremamente útil para o desenvolvimento tecnológico e industrial. Isso possibilita a identificação de possíveis problemas em etapas especificas da produção que comprometem o produto final, deixando-o fora de padrões pré-estabelecidos. A identificação e remoção de falhas durante a produção garantem não apenas produtos finais com melhor qualidade, mas também a diminuição de seus custos.

Para o CEP já foram propostos alguns gráficos construídos a partir de números fuzzy. Entretanto, seus autores salientam a necessidade de mais estudos na área (SENTÜRK et al., 2014; IOANNOU et al., 2002 e KUPONGSAK e TAN, 2006). No trabalho desenvolvido, os gráficos de controle de fuzzy foram menos rigorosos na avaliação da tendência central e um pouco mais rigorosos na avaliação da variabilidade para um grau de dominância mais rigoroso, quando comparados aos gráficos de controle de Shewhart. Essa diferença pode ser explicada pela construção do número fuzzy levar em consideração, além da centralidade das notas atribuídas pelos juízes, o grau de importância de cada nota e a variabilidade, o que não aconteceu nos gráficos de controle de Shewhart, onde as notas atribuídas pelos juízes são substituídas por um único valor, a média das notas. Outra vantagem dos gráficos de controle fuzzy é a criação de zonas de alerta, tanto internas quanto externas aos limites do gráfico, aumentando sua flexibilidade.

Então, no presente estudo, a interpretação feita pelo gráfico de controle fuzzy parece ser bastante adequada para ser usada na avaliação de processos onde os dados são obtidos por técnicas de mensuração de menor precisão. Entretanto, chama-se atenção para o fato de que no estudo de caso considerado não houve estabilização do processo. Outros estudos estão sendo desenvolvidos para analisar o desempenho dos gráficos aqui apresentados.

\section{REFERÊNCIAS}

ASMUS, T. C. ; DIMURO, G. P. . A total order for symmetric triangular (interval) fuzzy numbers. Mathware \& Soft Computing, v. 20, p. 76-85, 2013. 
BAAS, S. M.; KWAKERNAAK, $\mathrm{H}$. Rating and ranking of multiple-aspect alternatives using fuzzy sets. Automatica, v.13, p. 47-58, 1977. http://dx.doi.org/10.1016/00051098(77)90008-5

BARTLET, M. S.; KENDALL, D.G. The statistical analysis of Variance-Heterogeneity and the Logarithmic Transformation. Journal of the Royal Statistics Society, ser. B, v.8, p.128138, 1946. http://dx.doi.org/10.2307/2983618

BESTERFIELD, D. H. Control de calidad. 4 ed. Naucalpan de Juárez, México: Prentice Hall Hispano Americana, 1995.

BORTOLAN, G; DEGANI, R. A Review of some methods for ranking fuzzy subsets. Fuzzy Sets and Systems, v.15, p. 1-19, 1985. http://dx.doi.org/10.1016/0165-0114(85)90012-0

CHANG, W. Ranking of fuzzy utilities with triangular membership functions. In: INTERNATIONAL CONFERENCE ON POLICY ANALYSIS AND INFORMATION SYSTEMS, 1981. Taiwan, China. Anais...., Taiwan, 1981, p. 263-272.

CHEN, C. B.; KLEIN C. M. Fuzzy ranking methods for multi-attribute decision making. In: IEEE CONFERENCE SYSTEMS, MAN, AND CYBERNETICS, 1994. San Antonio, USA. Anais...., San Antonio, USA, 1994, v.1, p. 475-480.

CHEN, S. Ranking fuzzy numbers with maximizing set and minimizing set. Fuzzy Sets and Systems, v.17, p. 113-129, 1985. http://dx.doi.org/10.1016/0165-0114(85)90050-8

CHENG, Chi-Bin. Fuzzy process control: construction of control charts with fuzzy numbers. Fuzzy Sets and Systems, v.154, n.2, p.287-303, 2005.

http://dx.doi.org/10.1016/j.fss.2005.03.002

CIVILLE, G. V.; OFTEDAL, K. N. Sensory evaluation techniques - Make "good for you" taste "good". Physiology Behavior, n.107 (4), p. 598-605, 2012.

FRANCO, B. C.; CELANO, G. C.; CASTAGLIOLA, P.; COSTA, A. F. B. Economic design of Shewhart control charts for monitoring autocorrelated data with skip sampling strategies. International Journal of Production Economics, v. 151, p. 121-130, 2014. http://dx.doi.org/10.1016/j.ijpe.2014.02.008

IOANNOU, I.; PERROT, N.; HOSSENLOPP, J.; MAURIS, G.; TRYSTRAM, G. The fuzzy set theory: a helpful tool for the estimation of sensory properties of crusting sausage appearance by a single expert. Food Quality and Preference, v.13, p. 589-595, 2002.

http://dx.doi.org/10.1016/S0950-3293(02)00045-9

JOEKES, S.; SMREKAR, M.; BARBOSA, E. P. Extending a double sampling control chart for non-conforming proportion in high quality processes to the case of small samples. Statistical methodology, v. 23, p. 35-49, 2015. http://dx.doi.org/10.1016/j.stamet.2014.09.003

KUPONGSAK, S.; TAN, J. Application of fuzzy set and neural network techniques in determining food process control set points. Fuzzy Sets and Systems, v.157, p. 1169-1178, 2006. http://dx.doi.org/10.1016/i.fss.2005.12.015

MA, L. C.; Li, H. L. A fuzzy ranking method with range reduction techniques. European Journal of Operational Research, v.184, n.3, p.1032-1043, 2008.

http://dx.doi.org/10.1016/j.ejor.2006.12.023

MELLO, Carlos H. P. Gestão da qualidade. São Paulo: Person Education do Brasil, 2011. 
MONTGOMERY, D. C. Introdução ao controle estatístico da qualidade. 4.ed. Rio de Janeiro: LTC, 2012.

PINHO, A. F.; MONTEVECHI, J. A. B.; PAMPLONA, E. O. Aplicação de Números Fuzzy Triangulares em Análises de Investimentos em Situação de Incerteza - Método Baseado na Teoria dos Jogos. Pesquisa e Desenvolvimento Tecnológico, Itajubá, v. 21, n.2, p. $102-$ 107, 1997. Disponível em: http://www.iepg.unifei.edu.br/edson/download/Artpinhofuzzy.PDF. Acesso em: 03 set. 2014.

SAMOHYL, R. W. Controle estatístico da qualidade. Rio de Janeiro: Campus, 2009.

SENTÜRK, S.; ERGINEL, N.; KAYA, I.; KAHRAMAN, C. Fuzzy exponentially weighted moving average control chart for univariate data with a real case application. Applied Soft Computing, v. 22, p. 1-10, 2014. http://dx.doi.org/10.1016/j.asoc.2014.04.022

SILVA, L. M. T.; MEDEIROS, C. A. F.; COSTA, B. K. Qualidade dos serviços turísticos no setor de restaurantes: uma aplicação do modelo SERVPERF. Revista Hospitalidade. São Paulo, v. 6, n. 2, p. 115-139, 2009.

Shu, M. H.; Wu. H. C.: Fuzzy X and R control charts: Fuzzy dominance approach.

Computers \& Industrial Engineering, v. 61, n.3, p. 676-685, 2011.

Trip, A; Does, R. J. Quality quandaries: interpretation of signals from runs rules in Shewhart control charts. Quality Engineering, v. 22, n. 4, p. 351-357, 2010.

http://dx.doi.org/10.1080/08982112.2010.500190

YUAN, Y. Criteria for evaluating fuzzy ranking methods, Fuzzy Sets and Systems, v. 44, p. 139-157, 1991. http://dx.doi.org/10.1016/0165-0114(91)90073-Y

ZADEH, L. A. The concept of a Linguistic Variable and its Application to Approximate Reasoning, Information and Control - Parte I. Information Science, v. 8, p. 199-249, 1975. http://dx.doi.org/10.1016/0020-0255(75)90046-8

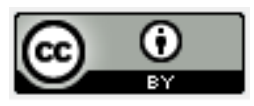

Artigo recebido em 10/11/2015 e aceito para publicação em 06/04/2016 DOI: http://dx.doi.org/ 10.14488/1676-1901.v16i2.2225 